\title{
IL-9 alterations linked to Alzheimer's disease in African Americans
}

Whitney Wharton, PhD ${ }^{1,2}$, Alexander L. Kollhoff, BS ${ }^{1}$, Umesh Gangishetti, PhD ${ }^{1}$, Danielle D. Verble, MA ${ }^{1}$, Samsara Upadhya, BS ${ }^{1}$, Henrik Zetterberg, PhD $^{3}$, Veena Kumar, MD ${ }^{1}$, Kelly D. Watts, MS ${ }^{1}$, Andrea J. Kippels, MSN ${ }^{1,2}$, Marla Gearing, PhD $^{2}$, J. Christina Howell, BS ${ }^{1,2}$, Monica W. Parker, MD ${ }^{1,2}$, William T. Hu, MD, PhD ${ }^{1,2}$

${ }^{1}$ Department of Neurology, Alzheimer's Disease Research Center, Emory University, Atlanta, USA.

${ }^{2}$ Alzheimer's Disease Research Center, Emory University, Atlanta, USA.

${ }^{3}$ UK Dementia Research Institute at UCL, London, UK.

\section{Abstract}

Objective-Compared to older Caucasians, older African Americans have higher risks of developing Alzheimer's disease (AD), and lower cerebrospinal fluid (CSF) tau biomarker levels. It is not known whether tau-related differences begin earlier in life, and whether race modifies other AD-related biomarkers such as inflammatory proteins.

Methods-We performed multiplex cytokine analysis in a healthy middle-aged cohort with family history of $\mathrm{AD}(\mathrm{n}=68)$ and an older cohort $(\mathrm{n}=125)$ with normal cognition $(\mathrm{NC})$, mild cognitive impairment (MCI), or AD dementia. After identifying baseline IL-9 level and ADassociated IL-9 change to differ according to race, we performed immunohistochemical analysis for proteins mechanistically linked to IL-9 in brains of African Americans and Caucasians ( $n=38)$, and analyzed post-mortem IL-9-related gene expression profiles in the publically available Mount Sinai cohort (26 African Americans and 180 Caucasians).

Results-Compared to Caucasians with NC, African Americans with NC had lower CSF tau, p$\mathrm{Tau}_{181}$, and IL-9 levels in both living cohorts. Conversely, AD was only correlated with increased CSF IL-9 levels in African Americans but not Caucasians. Immunohistochemical analysis revealed peri-vascular, neuronal, and glial cells immunoreactive to IL-9, and quantitative analysis in two independent US cohorts showed AD to correlate with molecular changes (upstream differentiation marker and downstream effector cell marker) of IL-9 upregulation only in African Americans but not Caucasians.

Corresponding author: William Hu, MD, PhD, Department of Neurology, 615 Michael Street, 505F, Atlanta, GA 30322, Phone 404-727-4174, Fax 404-727-3728, william.hu@emory.edu.

Author Contributions

WW, SU, KDW, MG, MWP, and WTH contributed to study concept and design; WW, ALK, UG, DDV, SU, HZ, VK, KDW, AJK, MG, JCH, MWP, and WTH contributed to data acquisition and analysis; WW, ALK, UG, DDV, SU, VK, JCH, MWP, and WTH contributed to drafting the manuscript and figures.

Potential conflicts of interests

Dr. Hu consults for ViveBio, LLC. which manufactures lumbar puncture trays. 
Interpretation-Baseline and AD-associated IL-9 differences between African Americans and Caucasians point to distinct molecular phenotypes for AD according to ancestry. Genetic and nongenetic factors need to be considered in future $\mathrm{AD}$ research involving unique populations.

\section{BACKGROUND}

Older African Americans are more than twice as likely to develop Alzheimer's disease (AD) dementia as older Caucasians. There are multiple potential causes for this, including the variable cognitive correlates for genetic AD risk factors, co-morbid cerebrovascular and systemic diseases, socioeconomic status, and psychosocial inequity. Clinicopathologic studies of $\mathrm{AD}$ in African Americans are rare due to low consent rates for autopsy, ${ }^{1}$ but available studies suggest greater prevalence of non-plaque/tangle co-pathology in African Americans. ${ }^{2,3}$ We and others have used antemortem biomarkers (CSF, MRI) to interrogate candidate mechanisms that may account for risk and phenotypic differences according to race. While some AD biomarkers (e.g., CSF beta-amyloid 1-42 or A $\beta 42$ ) exhibited the same alteration patterns in African Americans and Caucasians, other related biomarkers showed opposite directions of change (e.g., fronto-parietal functional connectivity) or attenuated changes in the same direction (e.g., CSF tau-related proteins). ${ }^{4}$ We thus hypothesize that, in addition to differential risks, older African Americans and Caucasians develop divergent downstream - pathologic and neuroprotective - processes upon cerebral amyloid deposition. However, these findings do not rule out aging-related differences, and here we sought to confirm previously-identified CSF biomarker differences in a middle-aged group of cognitively normal African Americans and Caucasians.

We also sought to identify inflammation-related biomarkers whose levels differ between the two races in middle- and older-age. Inflammation has been implicated in AD through genome-wide association, ${ }^{5-7}$ natural history, ${ }^{8-10}$ and neuropathologic studies. ${ }^{11-13}$ Race likely modifies baseline immune surveillance and inflammatory responses, as North ${ }^{14}$ and South ${ }^{15,16}$ Americans of African descent have more severe neuroinflammatory disorders (including multiple sclerosis, neuromyelitis optica, and idiopathic inflammatory disorders) than Caucasians with the same diagnoses. We previously found lower CSF levels of soluble vascular cell adhesion molecule 1 (sVCAM-1) in African Americans than Caucasians across the cognitive spectrum, which was counter-intuitive given the epidemiologic observation of more severe neuroinflammation. This can be potentially explained by elevated levels of other CSF cytokines. Alternatively, the relative change in CSF inflammatory biomarkers may better predict disease severity than their absolute levels. Increased CSF levels of chemokines and cytokines have were previously reported in $\mathrm{AD},{ }^{17,} 18$ the effect of race on the difference between normal cognition and $\mathrm{AD}$ has not been rigorously evaluated. Therefore, in addition to validating racial differences in CSF tau biomarker levels, we also examined race-dependent and race-independent CSF cytokine alterations. We then performed follow-up neuropathologic analyses in two autopsy cohorts, with a focus on IL-9 because its AD-related alteration was only seen in African Americans, and its expression is regulated by the transcriptional factor PU.1 encoded by the AD risk gene SPI1. ${ }^{19}$ 


\section{METHODS}

\section{Standard Protocol Approvals, Registrations, and Patient Consents}

The protocols were approved by the Emory University Institutional Review Boards and have therefore been performed in accordance with the ethical standards laid down in an appropriate version of the 1964 Declaration of Helsinki. Middle-aged African American and Caucasian subjects were recruited into a study of cognitively normal subjects with a family history of AD dementia (PI: Wharton), and older African American and Caucasian subjects were recruited into a study of normal cognition (NC), mild cognitive impairment (MCI), and AD dementia as previously described (PI: Hu). ${ }^{4}$ Written informed consents were obtained from all participants as well as their legal representatives when appropriate. The studies were registered on ClinicalTrials.gov under (Wharton) and (Hu).

\section{Subjects and pre-analytical processing}

Demographic (age, sex, education), diagnostic (syndrome, global Clinical Dementia Rating [CDR], Mini-Mental State Examination [MMSE]) and $A P O E$ allelic information were collected(Table 1). CSF (20 mL) was collected using protocols modified from the Alzheimer's Disease Neuroimaging Initiative (ADNI) ${ }^{20}$ using 24G Sprotte atraumatic needles and syringe between $8 \mathrm{AM}$ and noon without overnight fasting, and transferred into two $15 \mathrm{~mL}$ polypropylene tubes. For the middle-aged cohort, CSF was centrifuged at 2,000 rpm for cellular studies; the supernatant was removed, immediately aliquoted $(500 \mu \mathrm{L})$, labelled, and frozen $\left(-80^{\circ} \mathrm{C}\right)$ until analysis. For the older cohort, CSF was not centrifuged, and was immediately aliquoted $(500 \mu \mathrm{L})$, labeled, and frozen $\left(-80^{\circ} \mathrm{C}\right)$ until analysis. We previously prospectively determined that 2,000 rpm centrifugation did not influence cytokine levels measured here. ${ }^{21}$

\section{Subject grouping}

Each subject was categorized according to clinical diagnosis (NC, MCI, AD dementia; Table 1). Established CSF AD biomarkers (A $\beta 42$, total tau [t-Tau], and tau phosphorylated at threonine 181 [p-Tau $\left.\left.{ }_{181}\right]\right)$ were measured in the middle-aged cohort $(\mathrm{n}=68)$ using enzymelinked immunosorbent assays (Fujirebio, Ghent, Belgium) in the Zetterberg lab, and the same markers were previously measured in the older cohort using Luminex-based multiplex assays (Fujirebio US, Malvern, PA) in Atlanta. We have performed cross-platform validation studies at Emory to show strong linear correlation between these two platforms. ${ }^{22}$

\section{CSF cytokine \& chemokine assays}

Seven inflammatory proteins were selected for their preferential association with innate immunity or different immune cell populations (including T-helper [Th] cells 1, 2, 9, and 17): tumor necrosis alpha (TNF-a), macrophage-derived chemokine (MDC/CCL22), interleukin 7 (microglia), interleukin 8 (IL-8/CXCL8, Th17), interleukin 9 (IL-9, Th9), interleukin 10 (IL-10, Th2). Interferon gamma (Th1) levels were not consistently detectable. In its place, a downstream marker interferon gamma-induced protein (IP-10/CXCL10, Th1) was used as a surrogate. All these proteins were measured in a Luminex 200 platform using the Merck-Milliplex MAP Human Cytokine Panel (HCYTOMAG-60K, Merck-Millipore, 
Burlington, MA) following the manufacturer's protocol except two $100 \mu \mathrm{L}$ aliquots of CSF were used for duplicates. All operators were blinded to the diagnosis, and final assay results were then analyzed according to race, age, and diagnosis. In our laboratory, we achieve average intermediate precision (over experiments performed over 9 days) of 9.4\% for TNFa, $12.9 \%$ for MDC, $14.7 \%$ for IL-7, $4.8 \%$ for IP-10, $12.0 \%$ for IL-10, 9.2\% for IL-9, and 7.6\% for IL-8. Freeze-thawing experiments ${ }^{23}$ using CSF from 6 separate subjects showed significant degradation over two freeze-thaw cycles for MDC and TNF- $a(p=0.021$ and $\mathrm{p}=0.012$ for slope in exponential decay), and we previously showed light centrifugation ${ }^{21}$ to have minimal impact on these CSF cytokine levels.

\section{Immunohistochemistry}

To extend CSF-based differences in IL-9 to the brain, we performed immunohistochemistry (IHC) analysis on brains from 38 subjects (19 African Americans and 19 Caucasians; Table 2) from the Emory Alzheimer's Disease Research Center Brain Bank. African American cases were selected based on availability and exclusion of major non-AD pathology (e.g., intraparenchymal hemorrhage); Caucasian cases were age- and gender-matched to the African American cases. Both IHC and subsequent quantitative analysis were performed with the operators blinded to each case's race and diagnosis. Formalin-fixed superior middle frontal cortical sections were stained in the Lab-Vision Auto Stainer 480S platform (ThermoFisher) using primary antibodies targeting IL-9 (66144-1, EMD Millipore; 1:1500), CD3 (a marker for T-cells, ab5690, Abcam; 1:500), PU.1 (a known transcription for Th9 cells, E.388.3, ThermoFisher; 1:500), and tryptase (a mast cell marker related to Th9, M444905, EMD Millipore; 1:2000) followed by DAB-based detection. Slides were digitally scanned using the Aperio Digital Pathology Slide Scanner (Leica Biosystems, Buffalo Grove, IL). Quantitative pathologic analysis was performed using Aperio Image Scope (12.3.3.5048) to generate total area of positive staining. For each slide, a $100 \times 80$ grid was created for the coverslip to create $8,00050 \mu \mathrm{M} \times 50 \mu \mathrm{M}$ fields, and 25 fields were selected through randomly generated coordinates. A randomly selected field was analyzed using Aperio ImageScope if 1) more than half of the field was occupied by cortex and/or white matter; 2) there is no large mounting artifact (tear, fold); and 3) there is no significant staining artifact (e.g., DAB debris). Each case's mean immunoreactive area across 25 fields was then used for group-level statistical analysis.

\section{Statistical analysis}

Statistical analysis was performed by IBM-SPSS 24 (Armonk, NY) with effect sizes, 95\% confidence intervals $(\mathrm{CI})$, and p-values related to race, $\mathrm{AD}$, or their interaction (if significant) shown in Table 3. Model fit for the relationship between IL-9 and t-Tau (or p$\mathrm{Tau}_{181}$ ) was analyzed using Graph Pad Prism 8.0 (San Diego, CA). For baseline comparison among between the two races in the middle-aged group (all NC), Chi-squared tests (for categorical variables) and Student's T-tests (for continuous variables) were used with $p<0.01$ to adjust for multiple comparisons. For comparison between biomarker levels in this group, ANCOVA was first used to detect differences in established AD biomarkers (A $\beta 42$, t-Tau, p$\left.\mathrm{Tau}_{181}\right)$ and cytokines between races. All linear regression and ANCOVA were adjusted for age (age at death for the autopsy cohorts) and sex. Because some CSF cytokines correlate with tau biomarker levels, we also analyzed the relationship between cytokines and t-Tau (or 
p-Tau ${ }_{181}$ ) first through linear analysis, followed by the identification of possible inflection points through segmental linear regression (Prism 8.0) and iteratively selected inflection points.

CSF IL-9 levels were then analyzed in the older cohort of subjects with NC, MCI, and AD in relationship to race, $\mathrm{AD}$ diagnosis, cognition, and other Th-related cytokines. Student's Ttest was first used to determine whether CSF IL-9 levels differed between older African Americans and Caucasians with NC, and then between NC and AD subjects of the same race. Linear regression analysis was used to determine whether race modified IL-9 levels, the relationship between cognition and IL-9, or both. The same was completed for other cytokines (IP-10, IL-4, IL-10). The difference between race-normalized (through Ztransformation) IL-9 and IP-10 levels was finally calculated for each subject to derive a measure for Th1-to-Th9 bias, and linear regression was used to examine whether race modified the relationship between cognition and this bias.

For IHC analysis, ANCOVA was used to analyze areas of IL-9 and PU.1 immunoreactivity according to race and diagnosis (NC vs. AD). Mean brain IL-9-immunoreactive areas (pixels; averaged over 25 brain regions) were analyzed directly, and mean brain PU.1- and trypase-immunoreactive areas were $\log _{10}$-transformed because of non-normal distribution. An interaction term of race $\mathrm{x}$ diagnosis was introduced to determine whether AD-associated IL-9, $\log _{10}$ (PU.1), and $\log _{10}$ (tryptase) areas differed between the two racial groups.

For comparison with an independent cohort, the publically available proteomic and gene expression dataset from the Mount Sinai cohort (New York City, NY) was analyzed because of its inclusion of African Americans ( $\mathrm{n}=26)$ and Caucasians ( $\mathrm{n}=180)$ with NC, MCI, and $\mathrm{AD} .{ }^{24}$ Because detailed plaque density was available, we performed linear regression analysis to examine if race modified the relationship between neuritic plaque density and IL-9 related genes (including SPI1 for PU.1, IL4RA, IL33, TGFBR2, STAT6, SMAD3, and $O X 40$ genes associated with Th9 differentiation; and KIT as a mast cell receptor; IL 9 and $T P S A B 1$ [for mast cell tryptase beta] mRNA were not detected). As TGFBR2 and IL $4 R A$ expression levels were already log-transformed, the ratio of $T G F B R 2$ to $I L 4 R A$ was calculated by deriving the difference between log-transformed values.

\section{RESULTS}

\section{Race modified the relationship between CSF IL-9 and tau biomarkers}

We previously reported in the older cohort that African Americans had lower CSF t-Tau and p-Tau 181 levels than Caucasians within each diagnostic category. In a separate younger cohort (all with NC), analysis using a singleplex ELISA in an independent laboratory also showed lower CSF t-Tau (by 126 pg/mL, 95\% CI 51 - 201, p=0.001, Fig 1A) and p-Tau 181 (by $15.7 \mathrm{pg} / \mathrm{mL}, 95 \%$ CI $6.4-25.0, \mathrm{p}=0.003$, Fig 1B) levels in African Americans than Caucasians, adjusting for age and sex, despite similar A $\beta 42$ levels (by $17 \mathrm{pg} / \mathrm{mL}, 95 \% \mathrm{CI}$ $-87-120, \mathrm{p}=0.750$, Fig 1C).

In this middle-aged cohort, African Americans had lower CSF IL-7 (by $0.48 \mathrm{pg} / \mathrm{mL}, 95 \%$ CI $0.08-0.88, \mathrm{p}=0.021$ ) and IL-9 (by $1.11 \mathrm{pg} / \mathrm{mL}, 95 \%$ CI $0-2.22, \mathrm{p}=0.050$ ) levels than 
Caucasians. However, these findings may be mediated by a strong correlation between CSF p-Tau 181 and soluble cytokine levels which we have previously observed in neuroinflammatory conditions such as multiple sclerosis and HIV (Ozturk \& Hu, unpublished data). Thus, we next analyzed the relationship between race, tau markers, and IL-9. We found IL-9 to demonstrate non-linear relationships with both t-Tau and p-Tau ${ }_{181}$ in Caucasians (Fig 1D-E, top panels). Segmental linear analysis showed inflection points only for Caucasians (Fig 1D-E, lower panels), with similar relationships between the two races at low $\mathrm{t}$-Tau and $\mathrm{p}-\mathrm{Tau}_{181}$ concentrations but possible divergence at higher concentrations.

\section{AD dementia associated with higher CSF IL-9 levels in African Americans}

Even though CSF IL-9 levels appeared to plateau with greater tau markers in Caucasians with NC, there remains the possibility that IL-9 levels in African Americans may also plateau beyond a higher t-Tau or p-Tau 181 threshold. We thus next analyzed the effect of $\mathrm{AD}$ - associated with higher t-Tau and p-Tau 181 levels - on Th-9-related CSF proteins in a previously recruited older cohort of African Americans and Caucasians ${ }^{4}$ with $\mathrm{NC}, \mathrm{MCI}$, and AD. Older African Americans with NC also had lower CSF IL-9 levels than Caucasians with NC (by $1.90 \mathrm{pg} / \mathrm{mL}, 95 \% \mathrm{CI} 0.87-2.94, \mathrm{p}=0.001$ ), and AD diagnosis was only associated with greater CSF IL-9 levels in African Americans (by $1.27 \mathrm{pg} / \mathrm{mL}, 95 \%$ CI 0.35 - 2.19, $\mathrm{p}=0.010$ ). Analysis using a continuous measure of cognitive function (Fig $2 \mathrm{~B}$ ) controlling for age and sex also showed greater IL-9 in AD only in African Americans (increase of $0.552 \mathrm{pg} / \mathrm{mL}$ for each standard deviation of cognitive impairment, $95 \% \mathrm{CI}-0.076-1.179$, $\mathrm{p}=0.066$ ).

To better characterize cytokine alterations according to race and AD, we additionally analyzed CSF levels of IP-10, IL-4, and IL-10 associated with Th1, Th2, and Th2/Th9 pathways. AD dementia was associated with lower Th1-related IP-10 levels only in Caucasians (Fig 2C), but race did not significantly modify the effect of cognition when the latter was analyzed continuously (Fig 2D, decrease of $0.417 \mathrm{pg} / \mathrm{mL}$ for IP-10 for each standard deviation of cognitive impairment, $95 \%$ CI $0.015-0.828, \mathrm{p}=0.035)$. On the other hand, Th2-related IL-4 levels were too low for detection in this cohort, and Th2/Th9-related IL-10 levels did not differ according to race or cognition in the older cohort (consistent with previously reported U-shaped curve for IL-10). ${ }^{21}$ Thus, while AD was associated with increased Th9 activity only in African Americans, it may be only associated with decreased Th1 activity only in Caucasians.

To further test this hypothesis, it is useful to examine the relative level differences between cytokines of different pathways through normalization which we have found to control for pro-inflammatory changes associated with aging (i.e., inflammaging) within each individual. 25 To do this, we first transformed IL-9 and IP-10 into Z-scores according to a large cohort of cognitively normal adults recruited and analyzed at our university. We then derived a measure of IL-9 relative to IP-10 by subtracting Z-transformed IL-9 level by Z-transformed IP-10 level. This normalized measure then accounts for each cytokine's concentration ranges as well as factors which alter the two cytokines equally. This analysis revealed that, while race modified the relationship between these each cytokine and cognition, African Americans persistently had less Th1-to-Th9 bias than Caucasians (, even though this bias 
increased with progressive cognitive impairment (Figure 2E, 2F; slope of $0.212,95 \%$ CI $0.019-0.406, \mathrm{p}=0.018$ ).

\section{Race modified downstream, but not upstream, marker of IL-9 function}

Based on our findings so far, we hypothesized that African Americans had lower baseline brain IL-9 levels than Caucasians in the absence of AD pathology, but similar brain IL-9 levels as Caucasians in the setting of AD. To test this, we performed IHC analysis in an independent autopsy series of African Americans and Caucasians with NC or AD and identified two patterns of IL-9 staining in the brain (Table 2). Many cells on the albuminal side of small vessels were immunoreactive to IL-9 as well as the T-cell marker CD3, suggesting these to be peri-vascular Th9 cells (Fig 3A-C). Less intense IL-9 staining was identified in glia as well as neurons (Fig 3D), and these were not immunoreactive to CD3. Quantitative analysis of scanned slides taking into account both peri-vascular and parenchymal immunoreactivity showed African Americans with NC to have lower mean and median brain IL-9 levels than the other groups, but the large brain IL-9 variance required a much larger cohort to demonstrate statistical significance (Fig 4A).

IL-9 expression in neurons in a disease associated with neuronal loss may in part account for the wide variance we observed. We thus additionally examined up- and downstream markers of IL-9 functions. PU.1 is a critical transcription factor for Th9 differentiation, and we found AD to associated with elevated brain PU.1 regardless of race (Fig 3D \& 4B). In contrast, when we examined mast cells which represent major effectors of IL-9 in other organs, ${ }^{26,} 27$ we found $\mathrm{AD}$ to associate with higher brain tryptase levels only for African Americans but not Caucasians (Fig 3D \& 4C; $\mathrm{p}=0.063$ for race $\mathrm{X}$ diagnosis interaction).

\section{Brain IL4RA and KIT profiles in African Americans support Th9 polarization in AD}

Finally, we sought to replicate our findings in a separate clinicopathologic cohort of NC, $\mathrm{MCI}$, and AD cases. ${ }^{24}$ Proteomic analysis in the Mount Sinai cohort (26 African Americans, 180 Caucasians) did not detect IL-9 or any of proteins in its network, consistent with our and others' experience that only abundant proteins are reproducibly detected using an untargeted approach. ${ }^{28}$ Brain transcriptomics analysis on seven genes previously implicated in the IL-9 network showed SPI1 (encoding PU.1, p=0.030) to positively correlate with plaque burden independent of race, consistent with the brain IHC results from Emory for PU.1. When we examined two receptors (IL4RA,TGFBR2) linked to the differentiation of Th2 into Th9 cells, we found race to modify the relationship between plaque burden and IL $4 R A$ but not $T G F B R 2$ (which increased with plaque density independent of race, $\mathrm{p}=0.007$ ). Compared to Caucasians, African Americans had greater baseline IL4RA expression (Fig 5A, F(1, $202)=5.480, \mathrm{p}=0.020$ ), which decreased with greater neuritic plaque burden only in African Americans $(F(1,202)=5.441$ for race $X$ plaque burden, $\mathrm{p}=0.021)$.

Because an optimal combination (not necessarily equal concentration) of TGF- $\beta$ and IL-4 exposure is needed for in vitro Th9 differentiation but the in vivo ratio is not known, we hypothesized that $\mathrm{AD}$-associated $\mathrm{Th} 9$ differentiation was accompanied by an altered $T G F B R 2$-to- $I L 4 R A$ ratio. While this ratio was independent of plaque density in Caucasians, it increased linearly with greater plaque density in African Americans ( $\mathrm{p}=0.009$; Fig 5B). A 
nearly identical trend was observed when we examined expression of the mast cell receptor $K I T$ (Fig 5C). Taken together, we interpret these findings to associate a higher TGFBR2-to$I L 4 R A$ ratio with Th9 differentiation, which in turn enhances the activation of mast cells as a main effector of Th9 signaling.

\section{DISCUSSION}

The molecular basis for different clinical AD phenotypes between older African Americans and Caucasians is poorly understood. We previously identified lower CSF tau biomarker levels in older African Americans than older Caucasians regardless of diagnosis, and an opposite pattern of functional connectivity change involving brain areas perhaps more associated with tau than amyloid deposition. ${ }^{4}$ Here we extended our findings to a middleaged healthy cohort, and identified a new correlation between CSF IL-9 and AD-related changes only in African Americans. Furthermore, immunohistochemical and transcriptomic analysis of brain sections from two independent US cohorts revealed findings associated with Th9 differentiation and mast cell activation primarily in African Americans with AD. Taken together, we interpret these findings to support an AD-associated molecular phenotype switch between Th2 and Th9 in African Americans but not Caucasians.

IL-9 was previously thought to be released by Th2 cells, but is now recognized to originate from differentiated Th9 cells as well as Th17 cells and microvascular pericytes. ${ }^{29-31}$ The pleiotropic effects of IL-9 have been implicated in neurological (e.g., multiple sclerosis ${ }^{32,} 33$ and atopic myelitis ${ }^{34}$ ) as well as systemic (e.g., psoriasis ${ }^{35}$ and colitis ${ }^{36}$ ) disorders. IL-9 and its receptor have been found in neurons of the developing brain. ${ }^{37}$ To the best of our knowledge, this is the first report of neurons expressing IL-9 in mature brains. IL-9 is known to stimulate primary astrocytes and oligodendrocyte precursors without influencing microglial cells, ${ }^{38}$ but neurons can also themselves regulate T cells to release IL-9. ${ }^{39}$ Thus, IL-9 may represent a critical link in the cross-talk between neurons and peri-vascular T cells in the brain. What's more, IL-9 pathway was identified as a key point of genetic divergence between sub-Saharan Africans and African Americans, ${ }^{40}$ but it was not previously known how IL-9 levels varied between people of European vs. African ancestry. Changes related to IL-9 have not been consistently reported in $\mathrm{AD},{ }^{41,}{ }^{42}$ but there is intriguing animal data that knock-in $A P O E \varepsilon 4$ allele in mice elicits greater IL-9 production than the wildtype $\varepsilon 3$ allele. ${ }^{43}$ We did not observe an $A P O E$ effect on IL-9 levels in our series, but a larger cohort may be better powered to detect an interaction between these two factors.

We are not aware of a highly specific marker for Th9 cells as flow cytometry for Th9 cell has relied on marker/cytokine combinations (e.g., CD4 ${ }^{+}$IL-9 $9^{+}$IL-13-IFN- $\gamma^{-}$IL-17 $7^{-}$) not practical for large neuropathologic series. We thus examined multiple markers involved in Th9 differentiation and function in our neuropathologic analysis. In both racial groups, we found increased PU.1 transcript and protein levels in AD. This seems at first to contradict the race-specific changes in IL-9, but PU.1 is insufficient specific for Th9 and found in even higher levels in regulatory T cells. ${ }^{44}$ Similarly, TGFBR 2 - receptor for TGF- $\beta$ which regulates PU.1 expression also increased with AD regardless of race. TGF- $\beta$ binding to this receptor in the presence of IL-4 is necessary for Th9 differentiation, ${ }^{45}$ but the exact in vivo stoichiometry between TGF- $\beta$ and IL-4 is not known. We made the novel and important 
observation that $\mathrm{AD}$ was only associated with higher CSF IL-9 levels, higher brain TGFBR2/IL 4RA ratio, and higher brain mast cell markers in African Americans. We propose that a higher ratio of surface TGF- $\beta$ receptor to IL-4 receptor favors Th9 differentiation. Future experiments can confirm this hypothesis using single cell RNA-Seq analysis of CSF-derived T cells, as well as the clinically available IL-4 receptor blocker. ${ }^{46}$ If the observed alteration in IL-9 pathway proteins is related to slower cognitive decline observed in African Americans, ${ }^{47}$ manipulation of specific T cell lineages using a FDAapproved and well-tolerated agent may be an especially attractive therapy for AD in those without enhanced Th9 activity.

Our study was built on a large number of CSF samples from well-characterized middle-aged and older African Americans and Caucasians, IHC analysis in a second cohort, and transcriptomic analysis of a third cohort. It represents another step towards identifying biomarker and mechanism differences between individuals, but has some limitations. In keeping with reduced rates of participation in brain donation among US minority groups, ${ }^{48}$ the number of African Americans with available post-mortem tissue for analysis even if combined between the two cohorts is limited. We have not yet correlated CSF biomarker levels with the corresponding gene polymorphisms in the coding or regulatory regions, and we did not focus on Th9 cells during our initial CSF flow cytometry work. Our CSF cohort consists mostly of people from the southern US where there is the greatest concentration of African Americans, and it is not clear how well these findings will generalize to African Americans from other US regions or native Africans. IL-9 variance was much greater in the brain than in the CSF, possibly reflecting different pools (e.g., peri-vascular vs. neuronal) of IL-9 immunoreactive cells. IL 9 gene expression was itself not detected in the transcriptomic analysis, even though some of its network partners were. The uneven distribution, stability, detection, and variance for genes and proteins implicated in the same regulatory pathways are not unique to $\mathrm{AD}$ and neuroinflammation, and warrant caution in interpreting unsupervised analyses without consideration for each network member. We tried to overcome some of these challenges through multiple cohorts and techniques, and demonstrated the most convincing data to-date to support a different neuroinflammatory phenotype for $\mathrm{AD}$ in African Americans. While the exact causes genetic, environmental, cultural/behavioral and consequences for baseline and AD-related IL-9 differences between African Americans and Caucasians need further investigation, this and other similar studies reinforce the notion that not all AD-related changes derived in highly biased cohorts can be readily applied to real world populations.

\section{Acknowledgements}

The authors wish to acknowledge Allan I. Levey, MD, PhD, James J. Lah, MD, PhD, Chadwick Hales, MD, PhD, Jonathan D. Glass, MD, and James Newman, BS for collecting data and general support. This work was supported by National Institutes of Health [AG43885, AG42856, AG25688, K01AG042498].

\section{References}

1. Bonner GJ, Darkwa OK, Gorelick PB. Autopsy recruitment program for African Americans. Alzheimer Dis Assoc Disord. 2000 Oct-Dec;14(4):202-8. [PubMed: 11186597]

2. Barnes LL, Leurgans S, Aggarwal NT, et al. Mixed pathology is more likely in black than white decedents with Alzheimer dementia. Neurology. 20158 11;85(6):528-34. [PubMed: 26180136] 
3. Kamara DM, Gangishetti U, Gearing M, et al. Cerebral Amyloid Angiopathy: Similarity in AfricanAmericans and Caucasians with Alzheimer's Disease. J Alzheimers Dis. 2018;62(4):1815-26. [PubMed: 29614657]

4. Howell JC, Watts KD, Parker MW, et al. Race modifies the relationship between cognition and Alzheimer's disease cerebrospinal fluid biomarkers. Alzheimer's research \& therapy. 201711 2;9(1):88.

5. Guerreiro R, Wojtas A, Bras J, et al. TREM2 variants in Alzheimer's disease. N Engl J Med. 20131 10;368(2):117-27. [PubMed: 23150934]

6. Harold D, Abraham R, Hollingworth P, et al. Genome-wide association study identifies variants at CLU and PICALM associated with Alzheimer's disease. Nat Genet. 2009 10;41(10):1088-93. [PubMed: 19734902]

7. Lambert JC, Heath S, Even G, et al. Genome-wide association study identifies variants at CLU and CR1 associated with Alzheimer's disease. Nature genetics. 2009 10;41(10):1094-9. [PubMed: 19734903]

8. Andersen K, Launer LJ, Ott A, Hoes AW, Breteler MM, Hofman A. Do nonsteroidal antiinflammatory drugs decrease the risk for Alzheimer's disease? The Rotterdam Study. Neurology. 1995 8;45(8):1441-5. [PubMed: 7644037]

9. Beard CM, Waring SC, O'Brien PC, Kurland LT, Kokmen E. Nonsteroidal anti-inflammatory drug use and Alzheimer's disease: a case-control study in Rochester, Minnesota, 1980 through 1984. Mayo Clin Proc. 1998 10;73(10):951-5. [PubMed: 9787743]

10. Zandi PP, Anthony JC, Hayden KM, et al. Reduced incidence of AD with NSAID but not H2 receptor antagonists: the Cache County Study. Neurology. 20029 24;59(6):880-6. [PubMed: 12297571]

11. Veerhuis R, Janssen I, Hoozemans JJ, De Groot CJ, Hack CE, Eikelenboom P. Complement C1inhibitor expression in Alzheimer's disease. Acta Neuropathol. 1998 9;96(3):287-96. [PubMed: 9754962]

12. Sheng JG, Mrak RE, Griffin WS. Neuritic plaque evolution in Alzheimer's disease is accompanied by transition of activated microglia from primed to enlarged to phagocytic forms. Acta Neuropathol. 1997 7;94(1):1-5. [PubMed: 9224523]

13. Prokop S, Miller KR, Heppner FL. Microglia actions in Alzheimer's disease. Acta Neuropathol. 2013 10;126(4):461-77. [PubMed: 24224195]

14. Cree BA, Reich DE, Khan O, et al. Modification of Multiple Sclerosis Phenotypes by African Ancestry at HLA. Arch Neurol. 2009 2;66(2):226-33. [PubMed: 19204159]

15. Ferreira Vasconcelos CC, Santos Thuler LC, Cruz dos Santos GA, et al. Differences in the progression of primary progressive multiple sclerosis in Brazilians of African descent versus white Brazilian patients. Mult Scler. 2010 5;16(5):597-603. [PubMed: 20167593]

16. Papais-Alvarenga RM, Vasconcelos CC, Carra A, et al. Central Nervous System Idiopathic Inflammatory Demyelinating Disorders in South Americans: A Descriptive, Multicenter, CrossSectional Study. PLoS One. 2015;10(7):e0127757. [PubMed: 26222205]

17. Hu WT, Chen-Plotkin A, Arnold SE, et al. Novel CSF biomarkers for Alzheimer's disease and mild cognitive impairment. Acta neuropathologica. 2010 6;119(6):669-78. [PubMed: 20232070]

18. Craig-Schapiro R, Perrin RJ, Roe CM, et al. YKL-40: a novel prognostic fluid biomarker for preclinical Alzheimer's disease. Biological psychiatry. 201011 15;68(10):903-12. [PubMed: 21035623]

19. Huang KL, Marcora E, Pimenova AA, et al. A common haplotype lowers PU.1 expression in myeloid cells and delays onset of Alzheimer's disease. Nat Neurosci. 2017 8;20(8):1052-61. [PubMed: 28628103]

20. Shaw LM, Vanderstichele H, Knapik-Czajka M, et al. Cerebrospinal fluid biomarker signature in Alzheimer's disease neuroimaging initiative subjects. Annals of neurology. 2009 4;65(4):403-13. [PubMed: 19296504]

21. Gangishetti U, Christina Howell J, Perrin RJ, et al. Non-beta-amyloid/tau cerebrospinal fluid markers inform staging and progression in Alzheimer's disease. Alzheimers Res Ther. 20189 25;10(1):98. [PubMed: 30253800] 
22. Kollhoff AL, Howell JC, Hu WT. Automation vs. Experience: Measuring Alzheimer's BetaAmyloid 1-42 Peptide in the CSF. Front Aging Neurosci. 2018;10:253. [PubMed: 30186152]

23. Hu WT, Watts K, Grossman M, et al. Reduced CSF p-Tau181 to Tau ratio is a biomarker for FTLD-TDP. Neurology. 201311 26;81(22):1945-52. [PubMed: 24174584]

24. Wang M, Beckmann ND, Roussos P, et al. The Mount Sinai cohort of large-scale genomic, transcriptomic and proteomic data in Alzheimer's disease. Sci Data. 2018 9 11;5:180185. [PubMed: 30204156]

25. Giunta B, Fernandez F, Nikolic WV, et al. Inflammaging as a prodrome to Alzheimer's disease. J Neuroinflammation. 200811 11;5:51. [PubMed: 19014446]

26. Kearley J, Erjefalt JS, Andersson C, et al. IL-9 governs allergen-induced mast cell numbers in the lung and chronic remodeling of the airways. Am J Respir Crit Care Med. 20114 1;183(7):865-75. [PubMed: 20971830]

27. Forbes EE, Groschwitz K, Abonia JP, et al. IL-9- and mast cell-mediated intestinal permeability predisposes to oral antigen hypersensitivity. J Exp Med. 20084 14;205(4):897-913. [PubMed: 18378796]

28. Faria SS, Morris CF, Silva AR, et al. A Timely Shift from Shotgun to Targeted Proteomics and How It Can Be Groundbreaking for Cancer Research. Front Oncol. 2017;7:13. [PubMed: 28265552]

29. Schmitt E, Germann T, Goedert S, et al. IL-9 production of naive CD4+ T cells depends on IL-2, is synergistically enhanced by a combination of TGF-beta and IL-4, and is inhibited by IFN-gamma. J Immunol. 199411 1;153(9):3989-96. [PubMed: 7930607]

30. Veldhoen M, Uyttenhove C, van Snick J, et al. Transforming growth factor-beta 'reprograms' the differentiation of T helper 2 cells and promotes an interleukin 9-producing subset. Nature immunology. 2008 12;9(12):1341-6. [PubMed: 18931678]

31. Kovac A, Erickson MA, Banks WA. Brain microvascular pericytes are immunoactive in culture: cytokine, chemokine, nitric oxide, and LRP-1 expression in response to lipopolysaccharide. Journal of neuroinflammation. 201110 13;8:139. [PubMed: 21995440]

32. Nowak EC, Weaver CT, Turner H, et al. IL-9 as a mediator of Th17-driven inflammatory disease. The Journal of experimental medicine. 20098 3;206(8):1653-60. [PubMed: 19596803]

33. Li H, Nourbakhsh B, Ciric B, Zhang GX, Rostami A. Neutralization of IL-9 ameliorates experimental autoimmune encephalomyelitis by decreasing the effector $\mathrm{T}$ cell population. $\mathrm{J}$ Immunol. 201010 1;185(7):4095-100. [PubMed: 20805418]

34. Tanaka M, Matsushita T, Tateishi T, et al. Distinct CSF cytokine/chemokine profiles in atopic myelitis and other causes of myelitis. Neurology. 20089 23;71(13):974-81. [PubMed: 18809833]

35. Schlapbach C, Gehad A, Yang C, et al. Human TH9 cells are skin-tropic and have autocrine and paracrine proinflammatory capacity. Science translational medicine. 20141 15;6(219):219ra8.

36. Gerlach K, Hwang Y, Nikolaev A, et al. TH9 cells that express the transcription factor PU.1 drive T cell-mediated colitis via IL-9 receptor signaling in intestinal epithelial cells. Nature immunology. 2014 7;15(7):676-86. [PubMed: 24908389]

37. Fontaine RH, Cases O, Lelievre V, et al. IL-9/IL-9 receptor signaling selectively protects cortical neurons against developmental apoptosis. Cell death and differentiation. 2008 10;15(10):1542-52. [PubMed: 18551134]

38. Ding X, Cao F, Cui L, Ciric B, Zhang GX, Rostami A. IL-9 signaling affects central nervous system resident cells during inflammatory stimuli. Exp Mol Pathol. 2015 12;99(3):570-4. [PubMed: 26216406]

39. Liu Y, Teige I, Birnir B, Issazadeh-Navikas S. Neuron-mediated generation of regulatory T cells from encephalitogenic T cells suppresses EAE. Nat Med. 2006 5;12(5):518-25. [PubMed: 16633347]

40. Jin W, Xu S, Wang H, et al. Genome-wide detection of natural selection in African Americans preand post-admixture. Genome Res. 2012 3;22(3):519-27. [PubMed: 22128132]

41. Saresella M, Calabrese E, Marventano I, et al. Increased activity of Th-17 and Th-9 lymphocytes and a skewing of the post-thymic differentiation pathway are seen in Alzheimer's disease. Brain, behavior, and immunity. 2011 3;25(3):539-47. 
42. Stertz L, Contreras-Shannon V, Monroy-Jaramillo N, Sun J, Walss-Bass C. BACE1-Deficient Mice Exhibit Alterations in Immune System Pathways. Molecular neurobiology. 2018 1;55(1):709-17. [PubMed: 28004339]

43. Mace BE, Wang H, Lynch JR, et al. Apolipoprotein E modifies the CNS response to injury via a histamine-mediated pathway. Neurol Res. 2007 4;29(3):243-50. [PubMed: 17509222]

44. Goswami R, Jabeen R, Yagi R, et al. STAT6-dependent regulation of Th9 development. J Immunol. 20122 1;188(3):968-75. [PubMed: 22180613]

45. Takami M, Love RB, Iwashima M. TGF-beta converts apoptotic stimuli into the signal for Th9 differentiation. J Immunol. 20125 1;188(9):4369-75. [PubMed: 22461692]

46. Thaci D, Simpson EL, Beck LA, et al. Efficacy and safety of dupilumab in adults with moderateto-severe atopic dermatitis inadequately controlled by topical treatments: a randomised, placebocontrolled, dose-ranging phase 2b trial. Lancet. 2016 12;387(10013):40-52. [PubMed: 26454361]

47. Barnes LL, Wilson RS, Li Y, et al. Racial differences in the progression of cognitive decline in Alzheimer disease. Am J Geriatr Psychiatry. 2005 11;13(11):959-67. [PubMed: 16286439]

48. Jefferson AL, Lambe S, Cook E, Pimontel M, Palmisano J, Chaisson C. Factors associated with African American and White elders' participation in a brain donation program. Alzheimer Dis Assoc Disord. 2011 Jan-Mar;25(1):11-6. [PubMed: 20856099] 


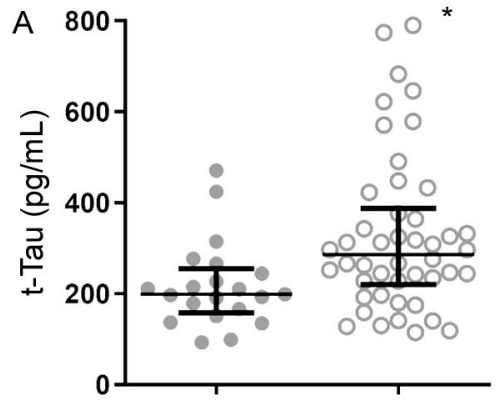

B

African

Americans

Figure 1.

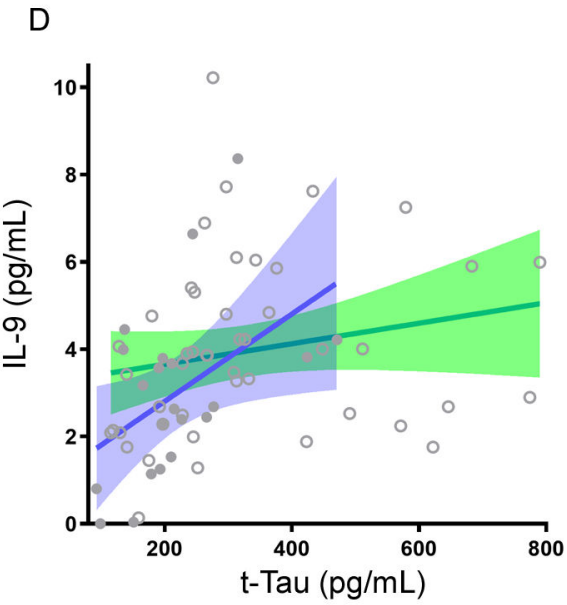

$\mathrm{E}$
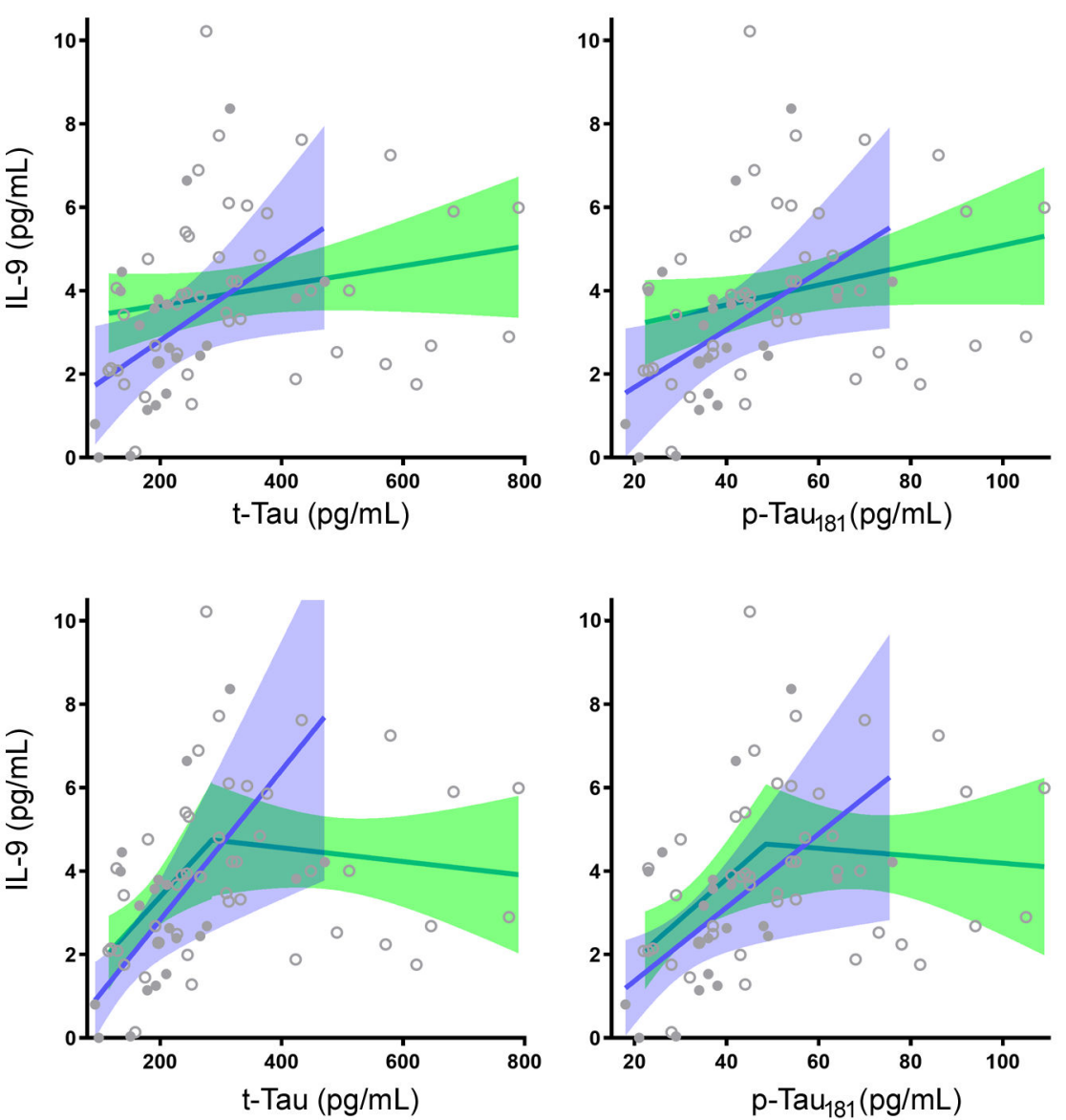

CSF AD biomarker and cytokine levels in middle-aged African Americans and Caucasians with normal cognition. Compared to Caucasians (open circles), African Americans (filled circles) had lower t-Tau (A, *) and p-Tau 181 (B, *) levels, but similar A 442 (C) levels. The relationship between CSF IL-9 and t-Tau (D) or p-Tau 181 (E) also differed according to race (top panels, blue lines for African Americans, green lines for Caucasians), and segmental linear analysis showed similar relationship between races at low t-Tau and p-Tau 181 concentrations but possible divergence at higher concentrations. Shaded areas represent 95\% $\mathrm{CI}$ of the regression curves. 

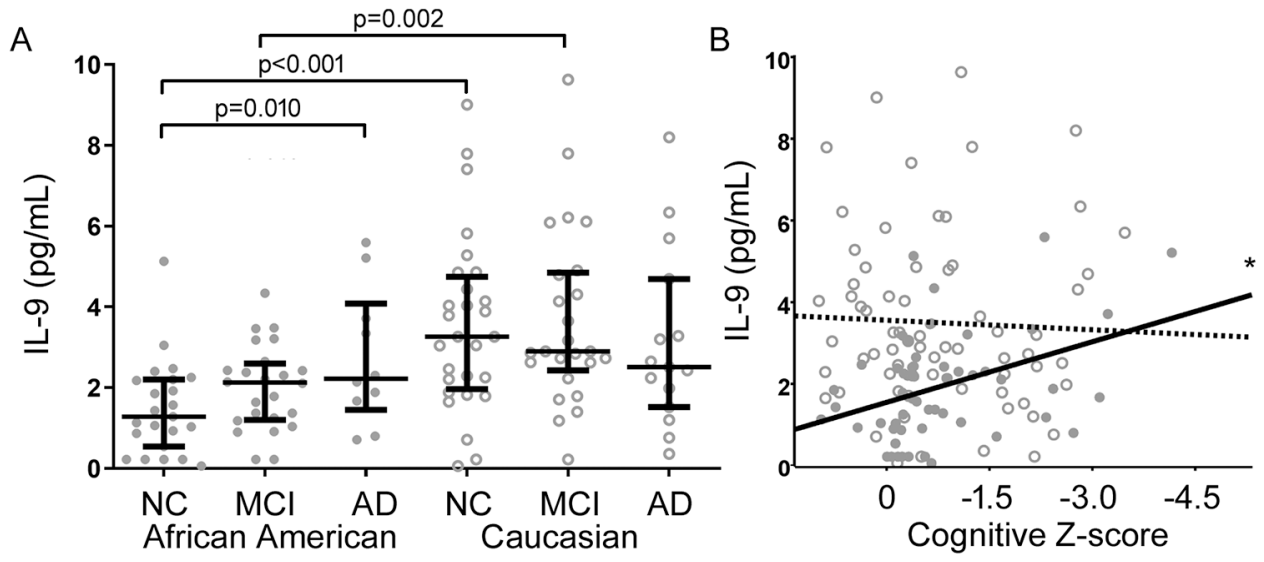

C

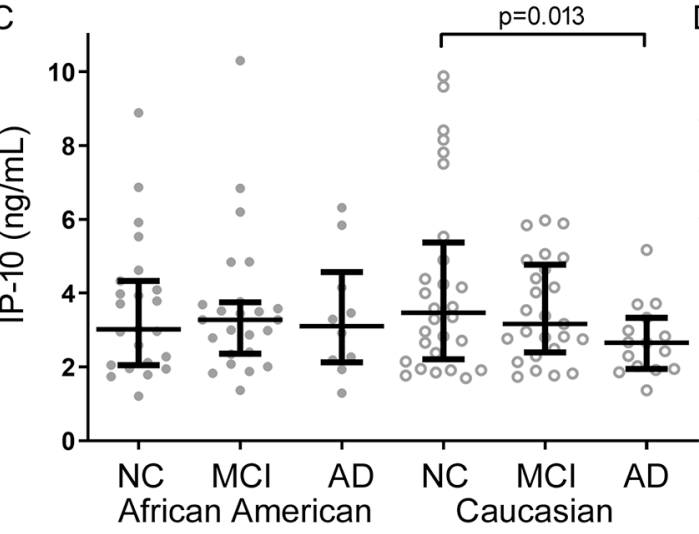

$\mathrm{D}$

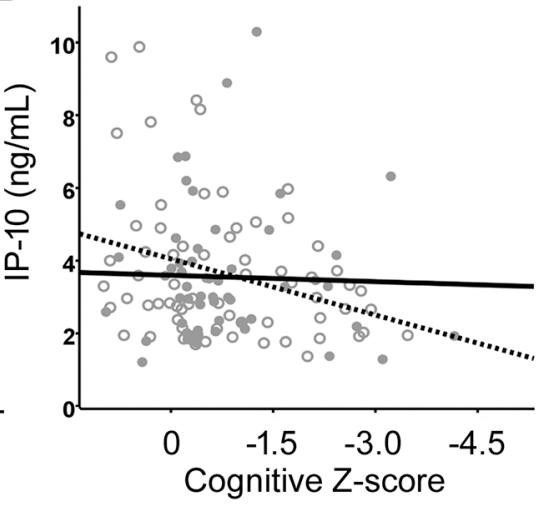

E

$\mathrm{F}$
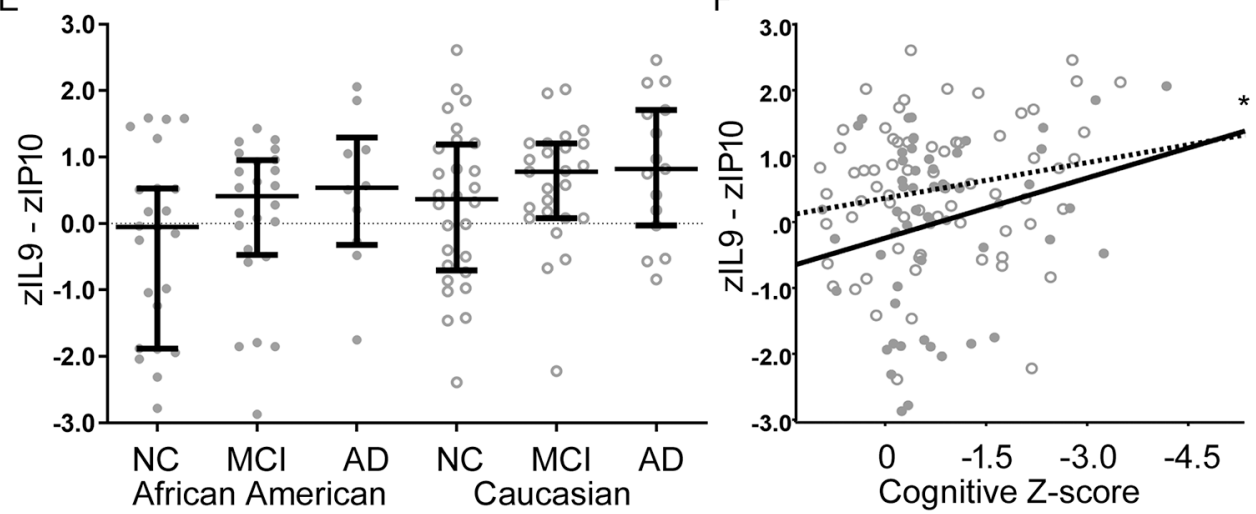

Figure 2.

CSF cytokine levels in older African Americans and Caucasians according to diagnosis (A, $\mathrm{C}, \mathrm{E})$ or a continuous measure of cognition (B, D, F). CSF IL-9 levels were lower in cognitively normal African Americans (closed circles) than Caucasians (open circles), and were associated with increases in MCI and AD dementia only in African Americans (A, B). Analysis of the balance between IL-9 and IP-10 showed similar AD-associated Th1-to-Th9 bias, with Caucasians showing a greater overall bias than African Americans. 

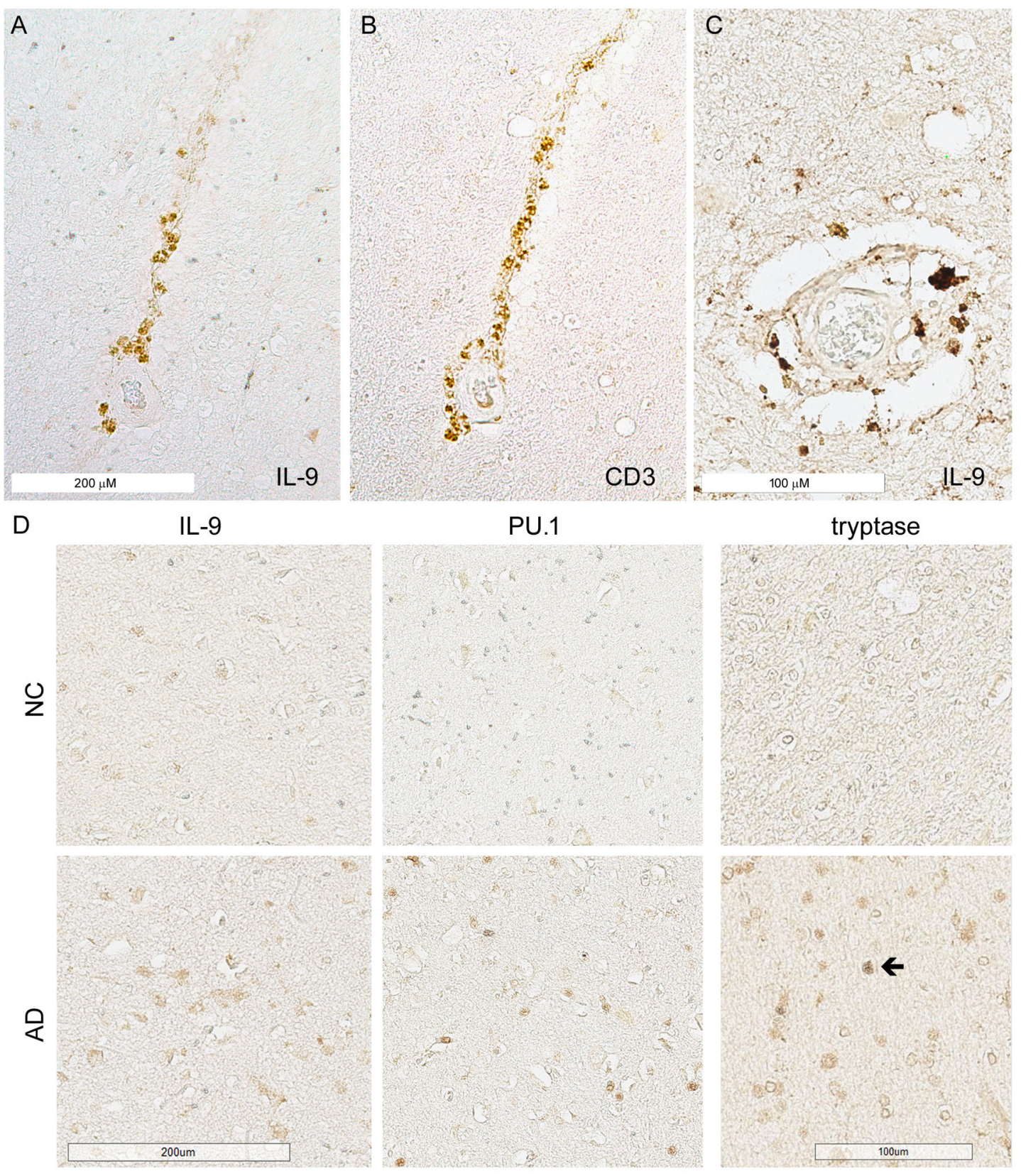

Figure 3.

IHC of protein markers related to Th9 in post-mortem brain tissue. In $\mathrm{AD}$, there was intense staining of IL-9 in perivascular cells (A) which are also immunoreactive to a T-cell marker CD3 (B) in adjacent slides suggesting these to be IL-9-containing T cells (higher magnification in C). There was also modest immunoreactivity to IL-9 in non-perivascular parenchymal neurons and glia (D), associated with increased PU.1 immunoreactivity (same magnification as IL-9) and strongly tryptase-positive cells (black arrow). 
A

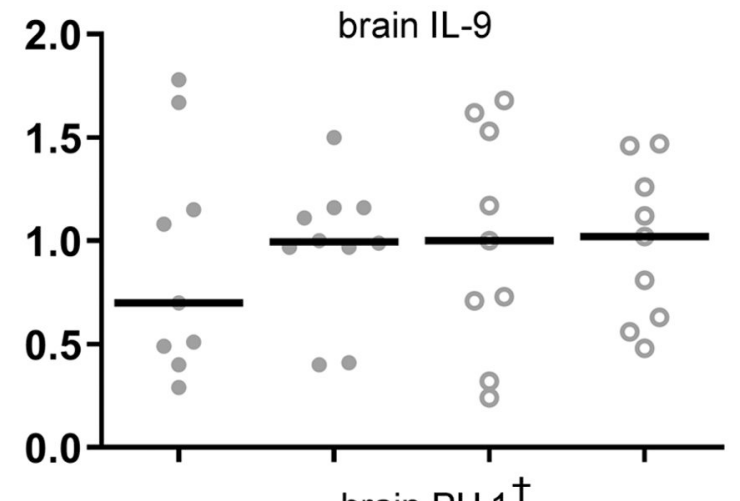

B

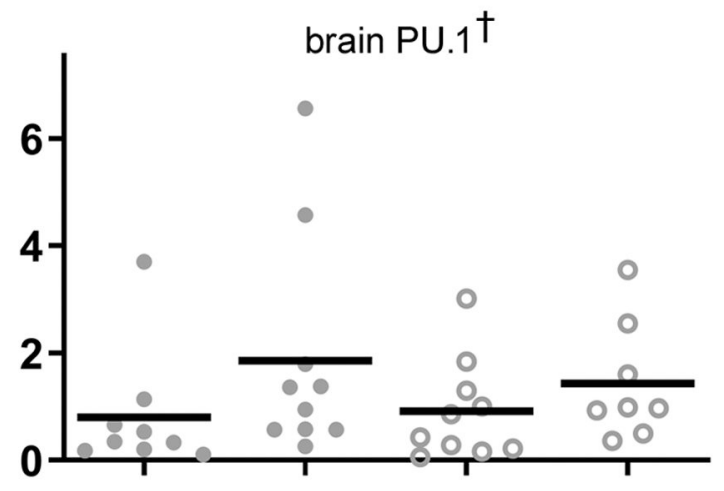

C

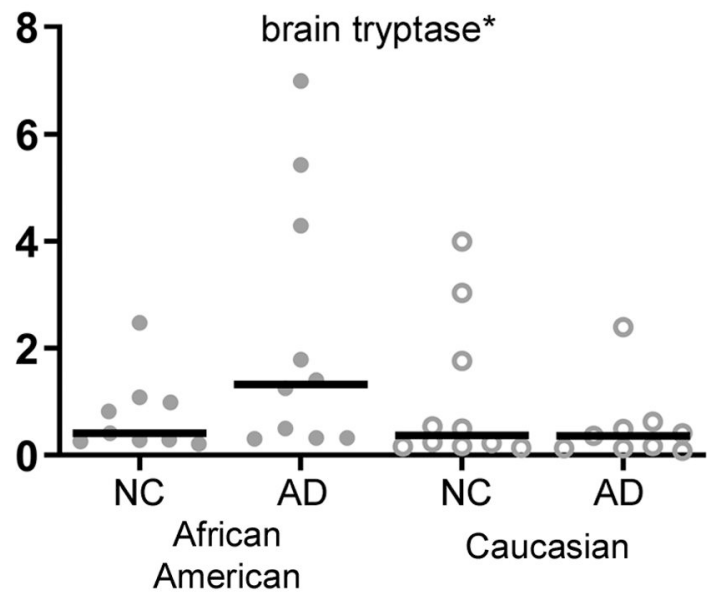

Figure 4.

Mean superior frontal IL-9 (A), PU.1 (B), and mast cell tryptase (C) immunoreactive areas in a post-mortem cohort from Emory. For each marker, immunoreactivity was normalized to the mean of Caucasian NC subjects. Greater PU.1 was associated with AD $(\dagger)$, while greater tryptase was only associated with African Americans with AD (*; bars represent median values. 

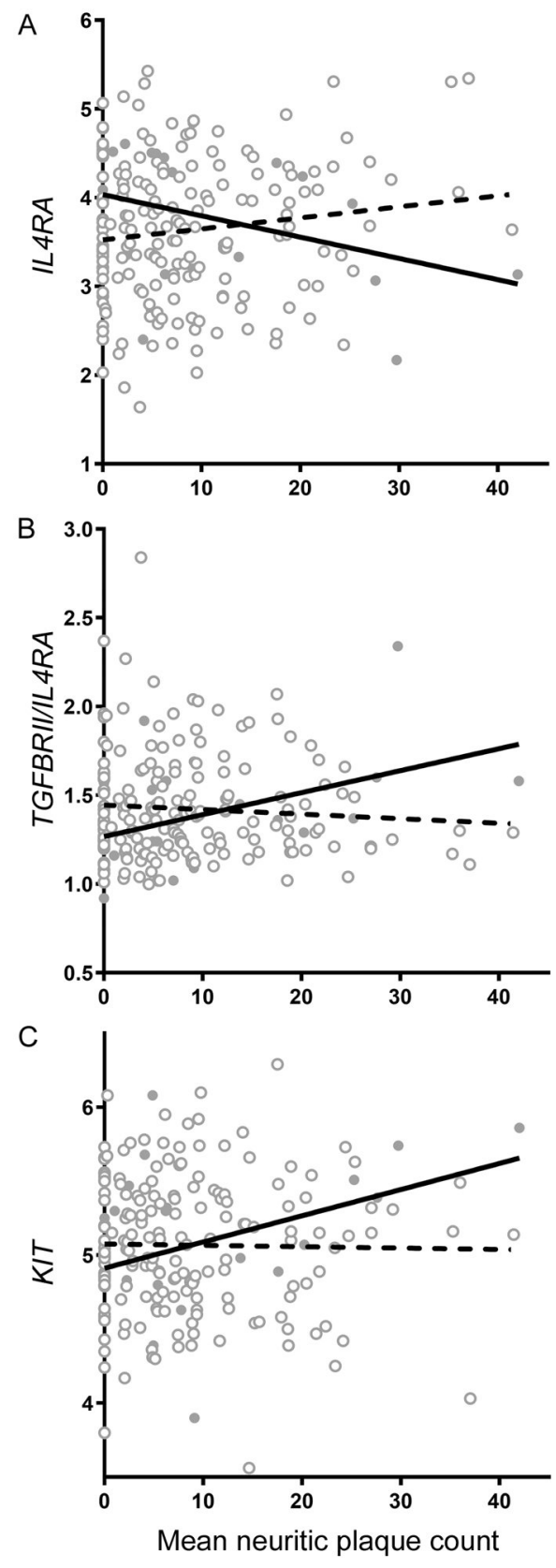

Figure 5.

Relationship between neuritic plaque density, race, and IL-9 related transcripts in the Mount Sinai cohort. For two genes involved in Th9 differentiation, race modified the relationship between plaque density and $I L 4 R A$ (A) but not TGFBR2 (Caucasians: open circles, dashed line; African Americans: filled circles, solid line). Analyzing the ratio of $T G F B R 2$ to $I L 4 R A$ ( $T G F B R 2 / I L 4 R A$ ) as a marker of Th9 differentiation showed a strong positive correlation with plaque density only in African Americans but not Caucasians (B). Examination of a mast cell (effector of Th9 cells) surface marker KIT/CD117 revealed the same changes as $T G F B R 2 / I L 4 R A$ ratio (C). 
Table 1.

Demographic features of subjects included in the current study (N.A.: not available).

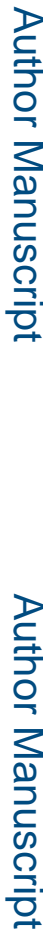

\begin{tabular}{|c|c|c|c|c|c|c|}
\hline & \multicolumn{2}{|c|}{$\begin{array}{l}\text { Middle-aged NC with } \\
\text { family history }\end{array}$} & \multicolumn{2}{|c|}{ Older NC } & \multicolumn{2}{|c|}{ MCI/AD } \\
\hline & $\underset{(\mathbf{n}=21)}{\operatorname{AfAm}}$ & $\begin{array}{c}\text { Cauc } \\
(\mathrm{n}=47)\end{array}$ & $\begin{array}{l}\text { AfAm } \\
(\mathbf{n}=23)\end{array}$ & $\begin{array}{c}\text { Cauc } \\
(\mathrm{n}=28)\end{array}$ & $\underset{(n=34)}{\operatorname{AfAm}}$ & $\begin{array}{c}\text { Cauc } \\
(n=40)\end{array}$ \\
\hline Male (\%) & $\begin{array}{c}4 \\
(19 \%)\end{array}$ & $\begin{array}{c}20 \\
(43 \%)\end{array}$ & $\begin{array}{c}9 \\
(39 \%)\end{array}$ & $\begin{array}{c}12 \\
(43 \%)\end{array}$ & $\begin{array}{c}17 \\
(50 \%)\end{array}$ & $\begin{array}{c}17 \\
(42 \%)\end{array}$ \\
\hline Age, yr (SD) & $\begin{array}{l}59.9 \\
(8.0)\end{array}$ & $\begin{array}{l}58.3 \\
(5.8)\end{array}$ & $\begin{array}{l}67.9 \\
(6.3)\end{array}$ & $\begin{array}{l}70.9 \\
(7.6)\end{array}$ & $\begin{array}{l}69.8 \\
(8.2)\end{array}$ & $\begin{array}{l}69.9 \\
(6.9)\end{array}$ \\
\hline Education, yr (SD) & N.A. & N.A. & $\begin{array}{l}16.2 \\
(2.5)\end{array}$ & $\begin{array}{l}17.0 \\
(2.6)\end{array}$ & $\begin{array}{l}16.5 \\
(2.9)\end{array}$ & $\begin{array}{l}16.0 \\
(2.7)\end{array}$ \\
\hline Having at least one APOE e4 allele & $\begin{array}{c}9 \\
(45 \%)\end{array}$ & $\begin{array}{c}24 \\
(53 \%)\end{array}$ & $\begin{array}{c}7 / 22 \\
(32 \%)\end{array}$ & $\begin{array}{c}11 \\
(39 \%)\end{array}$ & $\begin{array}{l}22 / 28 \\
(65 \%)\end{array}$ & $\begin{array}{c}24 \\
(60 \%)\end{array}$ \\
\hline TNF-a, pg/mL (SD) & $\begin{array}{c}1.30 \\
(0.94)\end{array}$ & $\begin{array}{l}1.11 \\
(0.81)\end{array}$ & $\begin{array}{c}1.98 \\
(0.73)\end{array}$ & $\begin{array}{l}2.33 \\
(0.85)\end{array}$ & $\begin{array}{c}2.18 \\
(0.79)\end{array}$ & $\begin{array}{c}2.04 \\
(0.74)\end{array}$ \\
\hline MDC, pg/mL (SD) & $\begin{array}{l}123.2 \\
(82.3)\end{array}$ & $\begin{array}{l}110.5 \\
(56.2)\end{array}$ & $\begin{array}{l}155.0 \\
(54.9)\end{array}$ & $\begin{array}{l}189.2 \\
(85.1)\end{array}$ & $\begin{array}{l}169.6 \\
(82.6)\end{array}$ & $\begin{array}{l}184.9 \\
(74.0)\end{array}$ \\
\hline IL-7, pg/mL (SD) & $\begin{array}{c}1.29 \\
(0.58)\end{array}$ & $\begin{array}{l}1.82 \\
(0.82)\end{array}$ & $\begin{array}{c}3.22 \\
(1.78)\end{array}$ & $\begin{array}{l}4.55 \\
(2.78)\end{array}$ & $\begin{array}{l}2.77 \\
(1.82)\end{array}$ & $\begin{array}{c}3.66 \\
(1.78)\end{array}$ \\
\hline IP-10, ng/mL (SD) & N.A. & N.A. & $\begin{array}{c}3.58 \\
(1.86)\end{array}$ & $\begin{array}{l}4.26 \\
(2.52)\end{array}$ & $\begin{array}{c}3.54 \\
(1.84)\end{array}$ & $\begin{array}{c}3.23 \\
(1.28)\end{array}$ \\
\hline IL-10, pg/mL (SD) & $\begin{array}{c}5.86 \\
(3.04)\end{array}$ & $\begin{array}{l}5.69 \\
(2.28)\end{array}$ & $\begin{array}{c}6.43 \\
(1.30)\end{array}$ & $\begin{array}{c}7.53 \\
(1.55)\end{array}$ & $\begin{array}{c}7.74 \\
(6.36)\end{array}$ & $\begin{array}{l}7.12 \\
(1.89)\end{array}$ \\
\hline IL-9, pg/mL (SD) & $\begin{array}{l}2.99 \\
(2.02)\end{array}$ & $\begin{array}{l}3.96 \\
(2.02)\end{array}$ & $\begin{array}{c}1.49 \\
(1.17)\end{array}$ & $\begin{array}{l}3.55 \\
(2.17)\end{array}$ & $\begin{array}{c}2.22 \\
(1.29)\end{array}$ & $\begin{array}{l}3.46 \\
(2.17)\end{array}$ \\
\hline IL-8, pg/mL (SD) & $\begin{array}{c}77.8 \\
(17.0)\end{array}$ & $\begin{array}{l}75.2 \\
(24.7)\end{array}$ & $\begin{array}{c}86.5 \\
(27.2)\end{array}$ & $\begin{array}{c}90.7 \\
(26.9)\end{array}$ & $\begin{array}{c}89.5 \\
(31.7)\end{array}$ & $\begin{array}{c}80.0 \\
(17.8)\end{array}$ \\
\hline
\end{tabular}

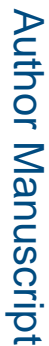

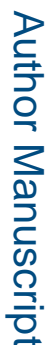


Table 2.

Demographic information for the Emory neuropathology cohort.

\begin{tabular}{|l|c|c|c|c|}
\hline & \multicolumn{2}{|c|}{ African Americans } & \multicolumn{2}{c|}{ Caucasians } \\
\hline & NC (n=9) & AD $(\mathbf{n = 1 0})$ & NC (n=10) & AD (n=9) \\
\hline Male (\%) & $4(44 \%)$ & $6(60 \%)$ & $5(50 \%)$ & $6(67 \%)$ \\
\hline Having at least one APOE ع4 allele & $3(33 \%)$ & $7(70 \%)$ & $1(10 \%)$ & $7(78 \%)$ \\
\hline Age at onset, yr (SD) & - & $55.5(12.8)$ & - & $58.7(10.2)$ \\
\hline Age at death, yr (SD) & $59.1(7.9)$ & $66.5(11.7)$ & $62.8(11.6)$ & $67.5(10.6)$ \\
\hline Mean IL-9, pixels (SD) & $107270(66137)$ & $115792(42314)$ & $119697(69392)$ & $117141(45278)$ \\
\hline Mean PU.1, pixels (SD) & $185388(263282)$ & $431646(478683)$ & $210630(228840)$ & $332307(254712)$ \\
\hline Mean tryptase, pixels (SD) & $592831(613032)$ & $1786394(1916010)$ & $850327(1098273)$ & $419912(569617)$ \\
\hline
\end{tabular}


Table 3.

Summary of relationships between race, CSF cytokines, and AD.

\begin{tabular}{|c|c|c|c|c|}
\hline Cohort & Outcomes & Factor & B $(95 \%$ CI $)$ & $\mathbf{p}$ \\
\hline \multirow[t]{2}{*}{ Middle-aged cohort with NC } & CSF IL-7 & AfAm & $-0.481(-0.885,-0.077)$ & 0.021 \\
\hline & CSF IL-9 & AfAm & $-1.109(-2.216,-0.002)$ & 0.050 \\
\hline \multirow[t]{7}{*}{ Older cohort with NC, MCI, AD } & CSF IL-9 & AfAm & $-1.981(-2.800,-1.162)$ & $<0.001$ \\
\hline & & Cognitive Z-scores & $0.064(0.338,0.466)$ & 0.753 \\
\hline & & AfAm X Cognitive Z-scores & $-0.552(-1.179,0.076)$ & 0.066 \\
\hline & CSF IP-10 & AfAm & $-0.262(-1.083,0.559)$ & 0.529 \\
\hline & & Cognitive Z-score & $0.417(0.015,0.828)$ & 0.035 \\
\hline & CSF zIL9-zIP10 & AfAm & $-0.537(-0.947,-0.126)$ & 0.015 \\
\hline & & Cognitive Z-scores & $-0.212(-0.406,-0.019)$ & 0.018 \\
\hline \multirow[t]{5}{*}{ Autopsy cohort 1} & PU.1 & AD & $0.079(0.018,0.140)$ & 0.015 \\
\hline & (III) & AfAm & $-0.014(-0.076,0.048)$ & 0.647 \\
\hline & Tryptase & $\mathrm{AD}$ & $-0.221(-0.670,0.229)$ & 0.326 \\
\hline & (III) & AfAm & $0.004(-0.446,0.454)$ & 0.986 \\
\hline & & AfAm X AD & $0.601(-0.035,1.237)$ & 0.063 \\
\hline \multirow[t]{11}{*}{ Autopsy cohort 2} & PU.1 & Plaque count & $0.011(0.001,0.021)$ & 0.030 \\
\hline & (III) & AfAm & $0.115(-0.150,0.381)$ & 0.392 \\
\hline & $I L 4 R A$ & Plaque count & $0.013(-0.001,0.027)$ & 0.066 \\
\hline & (III) & AfAm & $0.530(0.091,0.970)$ & 0.018 \\
\hline & & AfAm $X$ plaque count & $-0.037(-0.068,-0.006)$ & 0.021 \\
\hline & $T G F R 2 / I L 4 R A$ & Plaque count & $-0.003(-0.008,0.002)$ & 0.256 \\
\hline & (11 & AfAm & $-0.194(-0.360,-0.029)$ & 0.021 \\
\hline & & AfAm $X$ plaque count & $0.016(0.004,0.028)$ & 0.009 \\
\hline & $K I T$ & Plaque count & $-0.002(-0.010,0.007)$ & 0.716 \\
\hline & (IIIdast ceni) & AfAm & $-0.184(-0.453,0.086)$ & 0.181 \\
\hline & & AfAm X Plaque count & $0.018(0,0.037)$ & 0.056 \\
\hline
\end{tabular}

